\title{
Mild Encephalitis/Encephalopathy with Reversible Splenial Lesion (MERS) due to Acute Intermittent Porphyria with a novel mutation in the porphobinoglien deaminase gene
}

xiaoqing li

Peking Union Medical College Hospital

fei han

Peking Union Medical College Hospital qianlong chen

Chinese Academy of Medical Sciences \& Peking Union Medical College Fuwai Hospital tienan zhu

Peking Union Medical College Hospital yongqiang zhao

Peking Union Medical College Hospital

\section{xuezhong yu}

Chinese Academy of Medical Sciences and Peking Union Medical College

huadong zhu

Chinese Academy of Medical Sciences and Peking Union Medical College jian cao

Chinese Academy of Medical Sciences and Peking Union Medical College jing yang ( $\nabla$ yangbujing@126.com )

Peking Union Medical College Hospital https://orcid.org/0000-0002-8334-9034

Research

Keywords: acute porphyria, mild encephalopathy with a reversible splenial lesion, gene mutation, hyponatremia

Posted Date: January 3rd, 2020

DOI: https://doi.org/10.21203/rs.2.19929/v1

License: (a) This work is licensed under a Creative Commons Attribution 4.0 International License. Read Full License 


\section{Abstract}

Background: Mild encephalopathy with a reversible splenial lesion (MERS) is a clinico-radiological syndrome characterized by a reversible isolated lesion with transiently reduced diffusion in the central portion of the splenium of the corpus callosum (SCC). The reason for MERS is unknown. however, infectious-related MERS (in particular virus) remains the most common cause of reversible splenial lesions. Acute intermittent porphyria (AIP) is an autosomal dominant disorder caused by a partial deficiency of porphobilinogen deaminase (PBGD), the third enzyme in the of hemebiosynthetic pathway. It can affect the autonomic, peripheral, and central nervous system.

Result: In this study, we report a 20-year-old woman with AIP who presented with MR manifestations suggestive of MERS, she had a novel PBGD splicing mutation, a $\mathrm{G}$ to A mutation in base 594 resulting in tryptophan to a stop codon (W198*).

Conclusion: To the best of our knowledge, this is only one published case of MERS associated with AIP.

\section{Introduction}

Acute intermittent porphyria (AIP) is a rare autosomal dominant disorder affecting heme biosynthesis. AIP is caused by a partial deficiency of porphobilinogen deaminase (PBGD), with the alternative name hydroxymethylbilane synthase (HMBS), the third enzyme in the heme biosynthetic pathway. The presentation of AIP is highly variable and nonspecific and can involve the autonomic, peripheral and central nervous systems[1][2]. Mild encephalitis/encephalopathy with a reversible splenial lesion (MERS), first identified by Tada et al[3], is a clinico-radiological syndrome characterized by transient splenial lesions with high-signal-intensity on T2-weighted images (T2WI), fluid-attenuated inversion recovery images (FLAIR), and diffusion-weighted images (DWI); and hyper-isointense signals on T1-weighted imaging (T1WI) sequences without contrast enhancement[4]. The exact pathophysiology of MERS is unknown, to the best of our knowledge, there are no reports to date of reversible splenial lesions associated with AIP. Here we described an AIP case representing with MERS, which was confirmed the diagnosis with genetic testing of PBGD gene.

\section{Materials And Methods}

\section{Case report}

A 20-years-old Chinese Han woman, previously well, who came to hospital on 9 July 2019 with severe continuous abdominal pain, nausea, vomiting and dark tea-colored urine. she was diagnosed as having an "intestinal obstruction" and treated in a local hospital for a few days. Her serum sodium concentration was lower than normal $(121.9 \mathrm{mmol} / \mathrm{I} \downarrow)$. She became sleepiness, confusion and then convulsion on 12 July, On 13 July 2019, the patient was transferred to the emergency department of our hospital and her serum sodium concentration was decreased to $108 \mathrm{mmol} / \mathrm{L}$. Biochemical tests were positive for urine 
porphobilinogen (PBG), negative for free erythrocyte protoporphyrin and urine uroporphyrin, establishing the diagnosis of AIP. Her urine osmolality was normal $(119 \mathrm{mOsm} / \mathrm{kgH} 20)$ when plasma osmolality was lower than normal(249 mOsm/ $\mathrm{kgH} 20)$. However, due to the lack of hemin in China, only supportive treatments could be administered. After four days of treatment with $250 \mathrm{~g}$ of intravenous glucose per day and fluid restriction ( $\mathbb{2} 2000 \mathrm{ml}$ per day),with Tolvaptan $3.5 \mathrm{mg}$ once a day for 3 days, she recovered consciousness and her serum sodium concentration was gradually increased to $135 \mathrm{mmol} / \mathrm{L}$. Her initial brain magnetic resonance imaging on July 17 revealed an isolated lesion of the SCC, with T2 and FLAIR hyperintensity, T1 hypointensity, and corresponding reduced values on apparent diffusion coefficient (ADC) maps. (A, B and C of Fig. 1), whereas her cerebral spinal fluid (CSF) testing results were almost normal. The cranial MRI performed two weeks later revealed that the lesions determined on first MRI were significantly regressed (D, E and F of Fig. 1, respectively).

On the basis of these findings, we decided to examine the genetic causes of the disease in her family.

\section{Genetic testing of PBGD gene}

A written consent form, stating acceptance of genetic testing, was signed by the patient and his family members. This study was approved by the Ethics Committee of the Institutional Review Board at Peking Union Medical College Hospital (PUMCH). The molecular genetic test was performed by direct sequencing of the PBGD gene to confirm acute intermittent porphyria. All 14 exons of PBGD gene and a minimum of 20 base pairs of intronic DNA flanking of each exon were amplified by polymerase chain reaction (PCR) (Tiangen Biotech, Beijing, China) and subsequently sequenced through BigDye Terminator Cycle sequencing kit V 3.1 (ABI Biosystems) on an ABI PRISM 3730 Sequence Analyzer, according to the manufacturer's directions.

\section{Results}

A novel PBGD gene splicing mutation, c.594G >A (W198*), was detected by Sanger sequencing in the proband, which led to a premature termination codon (Fig. 2). After screening of her family members, we found that her mother also carried the same mutation, while her father did not (Fig. 2).

\section{Discussion}

MERS is a clinical-radiological syndrome with mild encephalitis/encephalopathy and the MRI finding of a transient lesion in SCC (type I MERS) or associated in other white matter areas (type II MERS). It has been reported that MERS is the second most common infectious encephalitis/encephalopathy syndrome in Japanese children[5] .

The syndrome mainly affects children and young adults; the prognosis is favorable with complete or nearly complete neurological and radiological resolution within days or weeks. The exact pathophysiology is unknown. There are few reports in the literature on MRI findings of porphyria cases with central nervous systems (CNS) involvement[6, 7]. Many of studies suggested that MRI changes are 
related to posterior reversible encephalopathy syndrome (PRES)[8-10]. In this report, we have presented a new MRI finding of AIP,which had never been reported before.

According to Hoshino et al[5],the diagnostic criteria of MERS are the following: (1) Clinical onset associated with neuropsychiatric symptoms, such as impaired consciousness within 1 week after fever onset; (2) Complete recovery without sequelae, mostly within 10 days after the onset of neuropsychiatric symptoms; (3) High-signal-intensity lesion in the SCC; (4) Involvement of the entire corpus callosum and bilateral cerebral white matter with symmetrical pattern may also occur; (5) Lesion disappearing within 1 week, with no residual signal changes or atrophy. Despite our proband performed the second MRI 15 days after the first one, and without fever, she fulfills this diagnostic criterion. MERS is classified in MERS type I and MERS type II, depending on the involvement of SCC alone or also other white matter areas, our patient was a MERS type I case.

Acute intermittent porphyria (AIP) is one of four forms of acute porphyria that is caused by an inherited deficiency of PBGD, which catalyzes the third enzymatic step in the biosynthesis of heme. Symptoms in AIP, which occur as intermittent attacks and may be life-threatening, are caused by excess production of porphyrin precursors on the visceral, peripheral, autonomic, and central nervous systems[1]. Clinical manifestations of CNS involvement include epileptic seizures, impaired consciousness, behavior changes and hyponatremia caused by inappropriate antidiuretic hormone syndrome[11, 12]. The symptoms of our proband included abdominal pain, nausea, vomiting, confusion, delirium, seizures and hyponatremia.

The reason for the transiently reduced diffusion within the lesions on MRI is still unknown, which has been suggested to be due to hypotonic hyponatremia or a myelin-specific neurotoxin released References by a pathogen[13,14]. The possible cause of hyponatremia of MERS is SIADH, which is also considered as a cause of hyponatremia in patients with AIP[12]. Since she had no evidence of infection, maybe hyponatremia as a contributing factor of MERS of our proband. However, urine and blood osmolality of our proband were both lower than normal, maybe because she had taken $0.25 \mathrm{mg}$ tolvaptan 12 hours before, tolvaptan could significantly decrease the urine osmolality $[15,16]$. Tolvaptan didn't exacerbating symptoms in the acute phase of AIP of our proband, maybe it is safe to use of Tolvaptan in AIP with hyponatremia.

In mainland of China, the lab investigations (erythrocytic PBG deaminase levels, urinary, fecal, and plasma porphyrin levels) are unavailable, molecular genetic testing provides a precise diagnosis to differentiate AIP from other acute porphyrias, and then can be used to identify AIP in relatives of the proband. In this study, we identified a novel PBGD gene mutation (W198*) in a Chinese family. Almost all cases of MERS have occurred in children in East Asian populations, mostly Japan, there has also been one case report of sisters with MERS, which would support the genetic vulnerability hypothesis[17]. Since AIP is a hereditary disease, suggesting a genetic factor might be involved in some MERS patients. This is the first reported case of MERS following AIP and she had a novel PBGD splicing mutation, our case report widens the phenotype of the neurological manifestations associated with AIP. Further clinical, radiological and genetic studies of MERS are necessary for a definite conclusion. 


\section{Conclusions}

In conclusion, we reported the first reported case of MERS following AIP with a novel PBGD splicing mutation. Maybe hyponatremia as a contributing factor of MERS.

\section{Abbreviations}

MERS®Mild Encephalitis/Encephalopathy with Reversible Splenial Lesion

AIP: Acute Intermittent Porphyria

PBGD冈porphobinoglien deaminase gene

SCC\splenium of the corpus callosum

HMBS \hydroxymethylbilane synthase

FLAIR 囚fluid-attenuated inversion recovery images

Ethics Approval and Consent to Participate

T2WI $₫ T 2$-weighted images

T1WI $₫ T 1$-weighted images

ADC 『apparent diffusion coefficient

CSF 『cerebral spinal fluid

PCR 『polymerase chain reaction

\section{Declarations}

Authors' Contributions

Xiaoqing Li and Jing Yang: substantial contributions to conception ,design and writing.

Fei Han and Jian Cao: MRI image analysis

Qianlong Chen: gene mutation detection

Tienan Zhu, Yongqiang Zhao, Xuezhong Yu, Huadong Zhu: all had drafting the article or revising it.

Acknowledgements

Not applicable. 
Funding

Funding for this research was supported by CAMS Fundamental research fund for Central public welfare research institute®2017PT31009®.

Ethics approval and consent to participate

All procedures followed were in accordance with the ethical standards of the responsible institutional committee on human experimentation and with the Helsinki Declaration of 1975 (revised in 2000). the Ethics Committee of the Institutional Review Board at Peking Union Medical College Hospital (PUMCH).A written consent form, stating acceptance of genetic testing, was signed by the patient and her family members.

Consent for publication

Written, informed consent was obtained from the patient's family.

Competing interests

The authors declare that they have no competing interests.

Availability of data and materials

The data used and/or analysed to support the results of the current study are available from the corresponding author on reasonable request.

\section{References}

1. Karim Z, Lyoumi S, Nicolas G, Deybach JC, Gouya L, Puy H: Porphyrias: A 2015 update. Clin Res Hepatol Gastroenterol 2015, 39(4):412-425.

2. Floderus Y, Shoolingin-Jordan PM, Harper P: Acute intermittent porphyria in Sweden. Molecular, functional and clinical consequences of some new mutations found in the porphobilinogen deaminase gene. Clin Genet 2002, 62(4):288-297.

3. Tada H, Takanashi J, Barkovich AJ, Oba H, Maeda M, Tsukahara H, Suzuki M, Yamamoto T, Shimono T, Ichiyama $\mathrm{T}$ et al: Clinically mild encephalitis/encephalopathy with a reversible splenial lesion. Neurology 2004, 63(10):1854-1858.

4. Cho JS, Ha SW, Han YS, Park SE, Hong KM, Han JH, Cho EK, Kim DE, Kim JG: Mild encephalopathy with reversible lesion in the splenium of the corpus callosum and bilateral frontal white matter. Journal of clinical neurology (Seoul, Korea) 2007, 3(1):53-56. 
5. Hoshino A, Saitoh M, Oka A, Okumura A, Kubota M, Saito Y, Takanashi J, Hirose S, Yamagata T, Yamanouchi $\mathrm{H}$ et al: Epidemiology of acute encephalopathy in Japan, with emphasis on the association of viruses and syndromes. Brain \& development 2012, 34(5):337-343.

6. Utz N, Kinkel B, Hedde JP, Bewermeyer H: MR imaging of acute intermittent porphyria mimicking reversible posterior leukoencephalopathy syndrome. Neuroradiology 2001, 43(12):1059-1062.

7. Aggarwal A, Quint DJ, Lynch JP: MR imaging of porphyric encephalopathy. AJR American journal of roentgenology 1994, 162(5):1218-1220.

8. Yang J, Yang H, Chen Q, Hua B, Zhu T, Zhao Y, Yu X, Zhu H, Zhou Z: Reversible MRI findings in a case of acute intermittent porphyria with a novel mutation in the porphobilinogen deaminase gene. Blood Cells Mol Dis 2017, 63:21-24.

9. Lambie D, Florkowski C, Sies C, Raizis A, Siu WK, Towns C: A case of hereditary coproporphyria with posterior reversible encephalopathy and novel coproporphyrinogen oxidase gene mutation c.863T>G (p.Leu288Trp). Annals of clinical biochemistry 2018, 55(5):616-619.

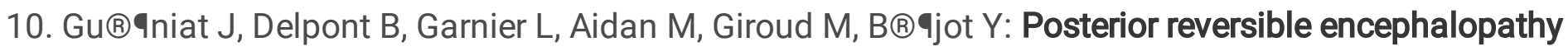
syndrome revealing acute intermittent porphyria. Revue neurologique 2016, 172(6-7):402-403.

11. Meyer UA, Schuurmans MM, Lindberg RL: Acute porphyrias: pathogenesis of neurological manifestations. Seminars in liver disease 1998, 18(1):43-52.

12. Yang J, Chen Q, Yang H, Hua B, Zhu T, Zhao Y, Zhu H, Yu X, Zhang L, Zhou Z: Clinical and Laboratory Features of Acute Porphyria: A Study of 36 Subjects in a Chinese Tertiary Referral Center. Biomed Res Int 2016, 2016:3927635.

13. Takanashi J, Tada H, Maeda M, Suzuki M, Terada H, Barkovich AJ: Encephalopathy with a reversible splenial lesion is associated with hyponatremia. Brain \& development 2009, 31(3):217-220.

14. Chen WX, Liu HS, Yang SD, Zeng SH, Gao YY, Du ZH, Li XJ, Lin HS, Liang HC, Mai JN: Reversible splenial lesion syndrome in children: Retrospective study and summary of case series. Brain \& development 2016, 38(10):915-927.

15. Devuyst O, Chapman AB, Gansevoort RT, Higashihara E, Perrone RD, Torres VE, Blais JD, Zhou W, Ouyang J, Czerwiec FS: Urine Osmolality, Response to Tolvaptan, and Outcome in Autosomal Dominant Polycystic Kidney Disease: Results from the TEMPO 3:4 Trial. Journal of the American Society of Nephrology : JASN 2017, 28(5):1592-1602.

16. Katsumata M, Hirawa N, Sumida K, Kagimoto M, Ehara Y, Okuyama Y, Fujita M, Fujiwara A, Kobayashi M, Kobayashi $Y$ et al: Effects of tolvaptan in patients with chronic kidney disease and chronic heart failure. Clinical and experimental nephrology 2017, 21(5):858-865.

17. Imamura T, Takanashi J, Yasugi J, Terada H, Nishimura A: Sisters with clinically mild encephalopathy with a reversible splenial lesion (MERS)-like features; Familial MERS? Journal of the neurological sciences 2010, 290(1-2):153-156. 
Figures
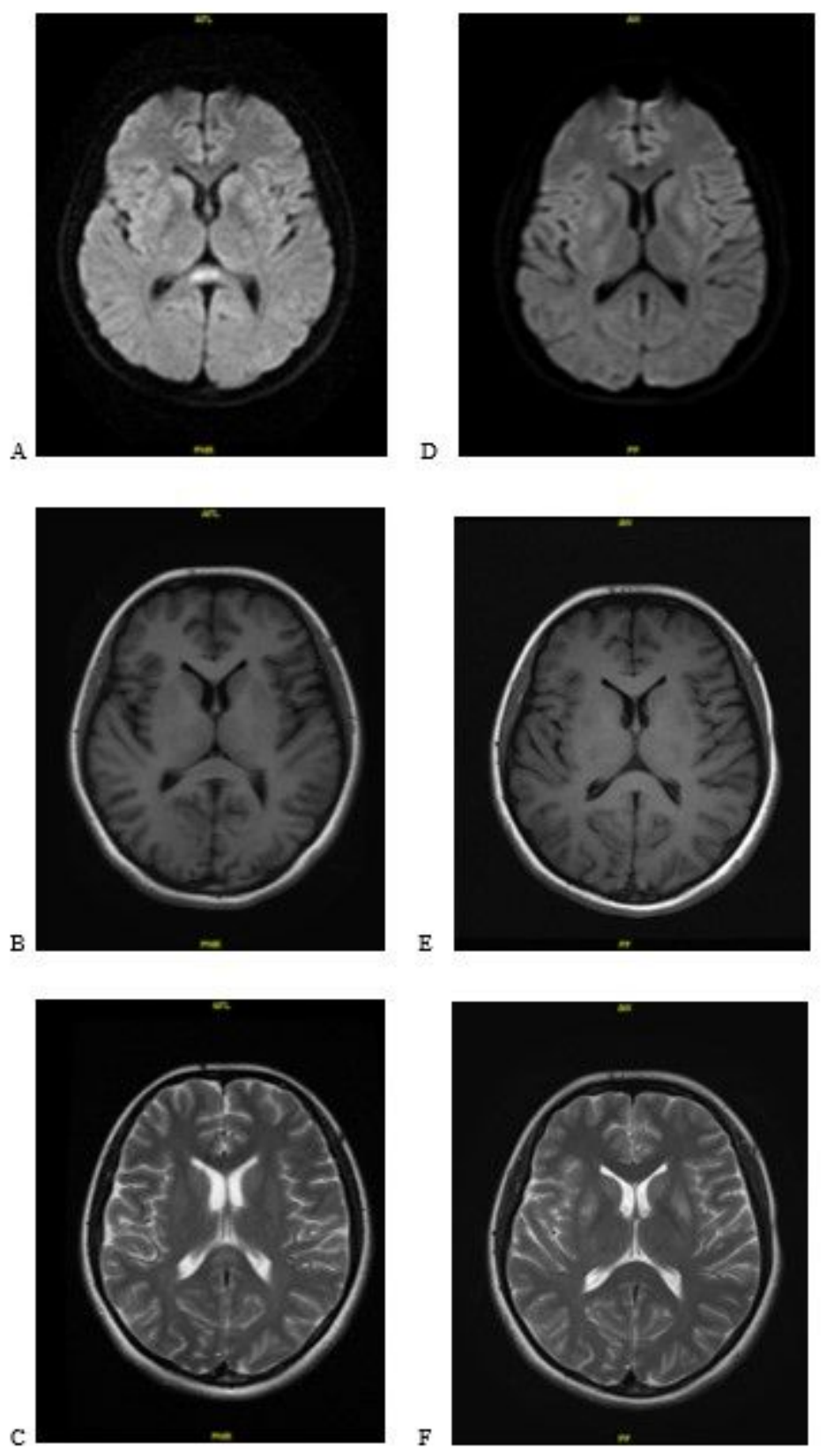

Figure 1

The lesion in the midline of SCC was hyperintensity on DWI (A) and T2WI (C), isointense signals on T1WI (B) (2019-7-17). Follow-up (2019-8-9) images show complete resolution(D,E,F). 
A.

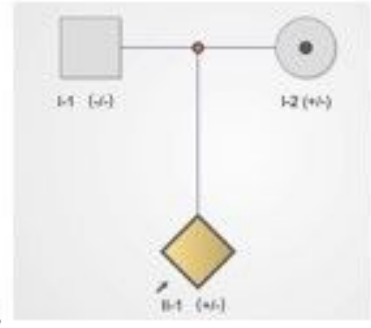

B.

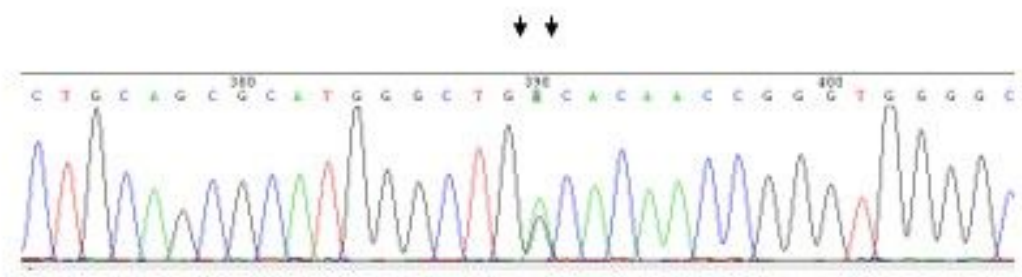

Figure 2

A novel PBGD gene frameshift mutation was identified. A. pedigree with PBGD gene mutation (The arrow indicated the proband); B. A novel PBGD gene mutation c.594G>A (W198*) was identified in the proband and her mother 